\title{
Violencia de parejas jóvenes a través de internet
}

\author{
BELÉN PIQUER BARRACHINA \\ al209517@uji.es \\ Jesús Castro Calvo \\ castroj@uji.es \\ CRISTINA GIMÉNEZ GARCíA \\ gimenezc@uji.es
}

\section{Resumen}

Introducción: El uso de internet amplía la cobertura en la que se puede ejercer la violencia de pareja entre los jóvenes. Por ello, el objetivo del presente estudio es analizar la prevalencia de la violencia entre parejas jóvenes a través de internet, examinando diferencias en función del género. Método: Los participantes son 109 jóvenes (44 chicos y 65 chicas) con una edad promedio de 22,86 años (DT $=2,859$ ), sin existir diferencias significativas en edad, orientación sexual y nivel de estudios, entre chicos y chicas. Los participantes cumplimentaron el Cyber Dating Abuse Questionnaire (Borrajo y cols., 2015) de manera confidencial y voluntaria. Resultados: Un 56,5 \% de la muestra reconoce controlar las actualizaciones de estado de sus parejas y el $40 \%$ asegura que sus parejas han empleado las nuevas tecnologías para saber dónde y con quién estaban, sin diferencias significativas por género. Según la prueba $t$ $(t=2,696 ; p \leq, 008)$, solo se establecen diferencias significativas, a favor de los chicos, en publicar contenidos para insultar o humillar a la pareja. Además, un $4,5 \%$ de chicos y chicas reconoce que podría ejercer algún tipo de abuso hacia su pareja, sin diferencias significativas de género. Conclusiones: Los resultados indican que entre esta población existen conductas que favorecen la exposición y realización de la violencia. En particular, el elemento de control prevalece en el uso de internet. Además, tanto chicos como chicas reconocen que podrían ejercer abuso hacia su pareja. Estos datos apoyan aquellos estudios que describen la violencia entre las parejas jóvenes cómo un fenómeno bidireccional.

Palabras clave: violencia, internet, jóvenes, género, pareja.

\section{Abstract}

Introduction: Internet use increases the possibilities of performing intimate partner violence among young people. Therefore, the objective of this study was to analyse prevalence of violence among young couples on the Internet by examining gender differences. Method: The sample included 109 young people (44 males, 65 females) whose average age was 22.86 years $(S D=2.859)$, with no significant gender differences in age, sexual orientation and level of education. Participants confidentially 
and voluntarily completed the Cyber Dating Abuse Questionnaire (Borrajo et al., 2015). Results: Of the sample, $56.5 \%$ reported the monitoring status updates on their partners' social networks, and $40 \%$ stated that their partners used new technologies to know where they were and who they were with. No significant gender differences were found. According to the Student's $t$-test $(t=2.696, p \leq .008)$, significant differences are observed for males related to posting content to insult and/or humiliate their partners. Moreover, $4.5 \%$ of males/females reported that they exerted some form of abuse on their partners, with no significant gender differences. Conclusion: These findings indicate behaviours among young people that encourage exposure and performing partner violence. In particular, the control element prevails in using new technologies. Both genders reported exerting abuse on partners. These data support studies that describe violence in young couples as a bidirectional phenomenon.

Keywords: violence, Internet, young people, gender, couples

\section{Introducción}

La violencia de pareja se ha reconocido en los últimos años como un problema social, convirtiéndose en el objetivo de numerosas investigaciones (Durán y Martínez-Pecino, 2015; Straus y Mickey, 2012). Parte de los estudios se han centrado más en la violencia que se da entre parejas adultas, en un contexto de convivencia, dejando de lado la que se puede producir en edades más tempranas y en situaciones de noviazgo. En cuanto a la perpetración y victimización de la violencia, clásicamente se ha visto que existen diferencias claras entre género, dándose tasas mayores de hombres que ejercen violencia y de mujeres víctimas de ella (Zweig y cols., 2013). Pero cabe destacar cómo según algunos autores (Fernández-González, 2013) esta regla cambia, encontrándose tasas similares de perpetración y victimización independientes del género. Así pues, en parejas jóvenes, el patrón de agresión podría darse de forma bidireccional (Straus y Luis, 2007; Straus y Mickey, 2012; Straus, 2004). Además, cuando se analiza la relación entre bidireccionalidad y edad, se encuentra que cuanto menor es la edad de los sujetos mayor es la bidireccionalidad y la prevalencia de agresión por parte de la mujer (Archer, 2002 en Fernández-González, 2013).

Por otro lado, las nuevas tecnologías amplían el campo en el que se puede ejercer violencia en las relaciones de pareja (Durán y Martínez-Pecino, 2015). Además, las personas jóvenes son consideradas nativas digitales. Por ejemplo, hasta el $75 \%$ de los jóvenes europeos usa internet (Living-Stone y Haddon, 2009).

La aparición de las nuevas tecnologías ha cambiado nuestra sociedad y, sobre todo, las relaciones que se establecen entre las personas. Internet y las redes sociales tienen un impacto positivo ya que proporcionan herramientas que facilitan la creación de relaciones interpersonales, el mantenimiento así como el aumento de la satisfacción con las mismas, pero también entrañan nuevos riesgos que antes eran inexistentes. Las nuevas tecnologías constituyen una nueva herramienta para el control y la vigilancia, haciendo que los jóvenes sean más accesibles y, por lo tanto, más susceptibles a la intrusión interpersonal y el acoso (Van Ouytsel, Van Gool, Walrave, Ponnet y Peeters, 2016). En el contexto de una relación de pareja, las redes sociales y las aplicaciones móviles propician situaciones de ciberacoso, control y abuso (Burke, Wallen, Vail-Smith y Knox, 2011; Durán y Martínez-Pecino, 2015; Van Ouytsel y cols., 2016; Zweig, 
Dank, Yahner y Lachman, 2013). Entendemos el ciberacoso como una forma de intimidación, acoso y malos tratos por parte de un individuo o grupo hacia otro, implicando el uso de las tecnologías como medio de agresión (Durán y Martínez-Pecino, 2015).

En cuanto a la prevalencia de la violencia de pareja en jóvenes, Durán y Martínez-Pecino (2015), en un estudio con población universitaria y edades comprendidas entre los 18 y 30 años, encontraron que un 57,2 \% declaraba haber sido víctima de acoso por parte de su pareja a través del teléfono móvil y un $27,4 \%$ a través de internet, siendo mayor el porcentaje de chicos victimizados. Además, un 47,6 \% declaró haber acosado a su pareja a través del teléfono móvil y un $14 \%$ a través de internet, siendo mayor el porcentaje de chicos acosadores. En otro estudio con población similar (Finn, 2004), se encontró que entre el $10 \%$ y el $15 \%$ habían sido víctimas de acoso por parte de sus parejas. Zweig y cols. (2013) encontraron que alrededor del $26 \%$ fueron víctimas de ciberacoso por parte de sus parejas en el último año, siendo la forma más frecuente de acoso el uso de las redes sociales sin permiso del encuestado.

En cuanto a las diferencias de género, las investigaciones no son concluyentes. Algunos autores afirman que los hombres son más victimizados que las mujeres (Durán y MartínezPecino, 2015), aunque estos ejerzan mayor acoso a sus parejas. En otro estudio (Zweig y cols., 2013), las mujeres reportaron mayores tasas de victimización con un $29 \%$ frente al $23 \%$ de los hombres. Por último, en una investigación realizada por Burke y cols. (2011), se observó que las mujeres ejercían un control y supervisión hacía sus parejas significativamente mayor que los hombres; concretamente, algunas mujeres comprobaban las redes sociales de sus parejas (más del $50 \%$ ), los correos electrónicos (20\%) y utilizaban las contraseñas de sus parejas (20\%) sin permiso para supervisar las conversaciones.

Los trabajos existentes respecto al ciberacoso han tendido a centrarse en población adolescente y contextos escolares, dejando al margen otros importantes grupos de edad, como los jóvenes, y otros contextos, como pueden ser las relaciones de noviazgo. Por ello, el objetivo del presente estudio es analizar la prevalencia de la violencia de pareja en jóvenes a través de internet, así como las diferencias de género que se puedan establecer.

\section{Método}

\section{Participantes}

La muestra está compuesta por un total de 109 jóvenes, con un rango de edad comprendido entre los 18 y 28 años ( $M=22,86$; DT = 2,859). De la muestra total, 65 eran chicas $(59,6 \%)$ y 44 eran chicos $(40,4 \%)$. En cuanto a la ocupación de los participantes, un 61,5\% eran estudiantes, el $28,4 \%$ se encontraban en activo y el $10,1 \%$ estaban en una situación de desempleo. El nivel de estudios más prevalente fue estudios universitarios $(49,5 \%)$, seguido de máster o doctorado $(20,2 \%)$ y bachillerato $(10,1 \%)$. Tan solo el 2,8 \% refirió no tener ningún tipo de estudio. En lo que se refiere a la nacionalidad, el 96,3\% de los participantes eran españoles y el $3,7 \%$ de otras nacionalidades. Por otro lado, se registraron datos sobre la orientación sexual, con 98 participantes $(89,9 \%)$ heterosexuales, $7(6,4 \%)$ homosexuales y 4 $(3,7 \%)$ bisexuales. Después de los análisis pertinentes se demostró que no existían diferencias significativas en edad $(t=-1,228 ; p \leq, 222)$, orientación sexual $(t=0,147 ; p \leq, 677)$ y nivel de estudios $(t=-1,598 ; p \leq, 113)$ entre chicos y chicas. 


\section{Procedimiento}

La obtención de los datos se realizó mediante un cuestionario online difundido a través de redes sociales y aplicaciones móviles. La información fue recogida entre enero y marzo del año 2016. La participación en el estudio fue totalmente anónima, confidencial y voluntaria. Antes de cumplimentar el instrumento, se informó a los participantes de la confidencialidad con la que se tratarían los datos y se obtuvo su consentimiento. Además, no se dotó de ningún tipo de remuneración económica.

\section{Instrumento de evaluación}

Para llevar a cabo el presente estudio se utilizó un cuestionario llamado Cyber Dating Abuse Questionnaire (CDAQ) (Borrajo y cols., 2015). Se trata de un instrumento compuesto por un total de 40 ítems en una escala tipo Likert de 6 puntos (nunca, no en el último año, raramente, a veces, frecuentemente, normalmente) que recogen información sobre distintos tipos de ciberacoso que se pueden dar en una relación de noviazgo como son: las amenazas, el robo de identidad, el control y la humillación. Existen 20 ítems donde se pide información acerca de la victimización y 20 ítems sobre la perpetración, es decir, la persona debe responder tanto si se lo hacen a ella como si ella lo realiza (por ejemplo, «Mi pareja o expareja ha creado un perfil falso de mí en una red social con el fin de causar problemas» o "He creado un perfil falso de mi pareja o expareja en una red social con el fin de causarle problemas»). Las instrucciones solicitan que señale las veces que la persona o su pareja/expareja han realizado alguna de las conductas durante el último año. En cuanto a la fiabilidad del instrumento, el $\alpha$ de Cronbach se encuentra entre ,73 y ,87 para las escalas que componen el instrumento (perpetración y victimización; control y agresión directa).

Por otra parte, también se recogió información de carácter demográfico: sexo, edad, nivel de estudios, ocupación y orientación sexual. A continuación, se pedía información sobre si alguna vez habían tenido pareja, si actualmente tenían pareja, el sexo de la pareja y la duración de la relación. Por último, se añadieron dos ítems sobre la percepción de riesgo ante la violencia de pareja y la posibilidad de ejercer abuso en una relación.

\section{Análisis de datos}

Se han realizado análisis descriptivos y análisis diferenciales (Chi-cuadrado y pruebas $t$ ). Los análisis estadísticos se realizaron mediante el programa SPSS statistics versión 23.

\section{Resultados}

Prevalencia de la perpetración y victimización en las relaciones de pareja y de los elementos de control y agresión directa

En lo que se refiere a la prevalencia de la victimización y la perpetración de la violencia, de los 109 jóvenes, un 18,63\% y un 18,23\% de chicos y chicas, respectivamente, han ejercido abuso hacia sus parejas a través de internet. Por otra parte, un $21,12 \%$ de chicos y un $15,52 \%$ de chicas se han sentido victimizados por sus parejas a través de internet. En cuanto a los datos más llamativos, cabe destacar que un 71,6 \% reconoce comprobar la hora de la última conexión de sus parejas en aplicaciones móviles o redes sociales, seguido de un $56,9 \%$ 
que controla las actualizaciones de estado de sus parejas. Así mismo, un 67,9 \% y un 51,4 \% asegura que sus parejas comprueban la hora de sus últimas conexiones y controlan sus actualizaciones de estado, respectivamente (véase la tabla 1).

En los ítems relacionados con el control, en la perpetración, los datos se sitúan entre el 15,6 \% («He llamado de forma excesiva a mi pareja o expareja para controlar dónde estaba y con quién») y el 71,6 \% («he comprobado la hora de la última conexión de mi pareja o expareja en aplicaciones móviles o redes sociales»). En la victimización, los datos en el elemento de control se sitúan entre el 17,4 \% («Me ha llamado de forma excesiva para controlar dónde estaba y con quién») y el 67,9\% («Ha comprobado la hora de mi última conexión en aplicaciones móviles o redes sociales»).

En los ítems relacionados con la agresión directa, tanto perpetración como victimización son menos prevalentes, aunque no por ello menos importantes; los datos en agresión directa en el factor victimización se sitúan entre el 0,9\% («Ha creado un perfil falso de mí en una red social con el fin de causar problemas») y el 9,2\% («Ha publicado música, poemas, frases, etc. en las actualizaciones de estado de su red social, refiriéndose a mí con la intención de insultarme y/o humillarme»). En el factor de perpetración las cifras se sitúan entre 0,9\% («He utilizado las nuevas tecnologías para hacerme pasar por mí pareja o expareja y causarle problemas») y el 8,3\% («He enviado mensajes a mi pareja o expareja insultándole y/o humillándole utilizando las nuevas tecnologías») (véase la tabla 1 ).

Tabla 1

Diferencias de género en CDAQ: perpetración y victimización de la violencia

\begin{tabular}{|c|c|c|c|c|}
\hline CDAQ & $\begin{array}{l}\text { Total } \\
(\%)\end{array}$ & $\begin{array}{c}\text { Chicos } \\
(\%)\end{array}$ & $\begin{array}{c}\text { Chicas } \\
(\%)\end{array}$ & $X^{2}$ \\
\hline $\begin{array}{l}\text { He comprobado la última conexión de mi pareja o expareja } \\
\text { en aplicaciones móviles o redes sociales. }\end{array}$ & 71,6 & 63,6 & 76,9 & 5,667 \\
\hline $\begin{array}{l}\text { Ha comprobado la hora de mi última conexión en aplica- } \\
\text { ciones móviles o redes sociales. }\end{array}$ & 67,9 & 72,7 & 64,6 & 9,654 \\
\hline $\begin{array}{l}\text { He controlado las actualizaciones de estado de mi pareja o } \\
\text { expareja. }\end{array}$ & 56,9 & 54,5 & 58,5 & 3,273 \\
\hline Ha controlado mis actualizaciones de estado en mi red social. & 51,4 & 50,0 & 52,3 & 6,613 \\
\hline $\begin{array}{l}\text { He controlado las amistades de mi pareja o expareja en } \\
\text { sus redes sociales. }\end{array}$ & 40,4 & 36,4 & 43,1 & 2,343 \\
\hline $\begin{array}{l}\text { Ha controlado las amistades que tengo en mis redes so- } \\
\text { ciales. }\end{array}$ & 34,9 & 40,9 & 30,8 & 6,821 \\
\hline $\begin{array}{l}\text { He revisado el teléfono móvil de mi pareja o expareja sin } \\
\text { su permiso. }\end{array}$ & 38,5 & 36,4 & 40,0 & 0,546 \\
\hline Ha revisado mi teléfono móvil sin mi permiso. & 33,9 & 36,4 & 32,3 & 2,701 \\
\hline $\begin{array}{l}\text { He revisado las redes sociales, WhatsApp o el correo elec- } \\
\text { trónico de mi pareja o expareja sin su permiso. }\end{array}$ & 34,9 & 27,3 & 40,0 & 5,713 \\
\hline $\begin{array}{l}\text { Ha revisado mis redes sociales, WhatsApp o mi correo } \\
\text { electrónico sin mi permiso. }\end{array}$ & 25,7 & 31,8 & 21,5 & 6,565 \\
\hline $\begin{array}{l}\text { He utilizado las nuevas tecnologías para controlar dónde y } \\
\text { con quién ha estado mi pareja o expareja. }\end{array}$ & 32,1 & 38,6 & 27,7 & 8,877 \\
\hline
\end{tabular}




\begin{tabular}{|c|c|c|c|c|}
\hline CDAQ & $\begin{array}{l}\text { Total } \\
(\%)\end{array}$ & $\begin{array}{c}\text { Chicos } \\
(\%)\end{array}$ & $\begin{array}{l}\text { Chicas } \\
(\%)\end{array}$ & $X^{2}$ \\
\hline $\begin{array}{l}\text { Ha utilizado las nuevas tecnologías para controlar dónde y } \\
\text { con quién he estado. }\end{array}$ & 33,0 & 36,4 & 30,8 & 4,825 \\
\hline $\begin{array}{l}\text { He exigido a mi pareja o expareja que responda inmedia- } \\
\text { tamente a mis llamadas o mensajes. }\end{array}$ & 30,3 & 38,6 & 24,6 & 5,528 \\
\hline $\begin{array}{l}\text { Me ha exigido que responda a sus llamadas o mensajes } \\
\text { inmediatamente. }\end{array}$ & 36,7 & 43,2 & 32,3 & 1,494 \\
\hline $\begin{array}{l}\text { He utilizado las contraseñas, sin permiso, para examinar } \\
\text { los mensajes o contactos de mi pareja o expareja. }\end{array}$ & 23,9 & 22,7 & 24,6 & 1,047 \\
\hline $\begin{array}{l}\text { Ha utilizado mis contraseñas, sin permiso, para examinar } \\
\text { mis mensajes o contactos. }\end{array}$ & 19,3 & 22,5 & 16,9 & 3,958 \\
\hline $\begin{array}{l}\text { He llamado de forma excesiva a mi pareja o expareja para } \\
\text { controlar dónde estaba y con quién. }\end{array}$ & 15,6 & 22,7 & 10,8 & 4,031 \\
\hline $\begin{array}{l}\text { Me ha llamado de forma excesiva para controlar dónde } \\
\text { estaba y con quién. }\end{array}$ & 17,4 & 25,0 & 12,3 & 4,176 \\
\hline $\begin{array}{l}\text { He enviado mensajes a mi pareja o expareja insultándole } \\
\text { y/o humillándole. }\end{array}$ & 8,3 & 9,1 & 7,7 & 4,314 \\
\hline $\begin{array}{l}\text { Me ha enviado mensajes insultándome y/o humillándome } \\
\text { a través de las nuevas tecnologías. }\end{array}$ & 6,4 & 9,1 & 4,6 & 7,996 \\
\hline $\begin{array}{l}\text { He publicado frases, etc. en las actualizaciones de estado, } \\
\text { refiriéndome a mi pareja o expareja con la intención de } \\
\text { insultar y/o humillar. }\end{array}$ & 4,6 & 11,4 & 0,0 & 7,741 \\
\hline $\begin{array}{l}\text { Ha publicado frases, etc. en las actualizaciones de estado, } \\
\text { refiriéndose a mí con la intención de insultar y/o humillar. }\end{array}$ & 9,2 & 15,9 & 4,6 & 4,432 \\
\hline $\begin{array}{l}\text { Me he hecho pasar por otra persona para poner a prueba } \\
\text { a mi pareja o expareja. }\end{array}$ & 3,7 & 0,0 & 6,2 & 2,811 \\
\hline Se ha hecho pasar por otra persona para ponerme a prueba. & 2,8 & 4,5 & 1,5 & 3,656 \\
\hline $\begin{array}{l}\text { He difundido rumores, chismes y/o mentiras sobre mi pa- } \\
\text { reja o expareja, con la intención de ridiculizarle. }\end{array}$ & 1,8 & 4,5 & 0,0 & 3,010 \\
\hline $\begin{array}{l}\text { Ha difundido rumores, chismes y/o mentiras sobre mí, con } \\
\text { la intención de ridiculizarme. }\end{array}$ & 3,7 & 6,8 & 1,5 & 3,108 \\
\hline $\begin{array}{l}\text { He revelado secretos o información comprometida sobre } \\
\text { mi pareja o expareja. }\end{array}$ & 1,8 & 0,0 & 3,1 & 1,379 \\
\hline Ha revelado secretos o información comprometida sobre mí. & 1,8 & 4,5 & 0,0 & 3,010 \\
\hline $\begin{array}{l}\text { He amenazado a mi pareja o expareja con revelar secre- } \\
\text { tos o información comprometida sobre ella. }\end{array}$ & 1,8 & 2,3 & 1,5 & 2,156 \\
\hline $\begin{array}{l}\text { Ha amenazado con revelar secretos o información com- } \\
\text { prometida sobre mí. }\end{array}$ & 2,8 & 6,8 & 0,0 & 4,557 \\
\hline $\begin{array}{l}\text { He escrito un comentario en el muro de una red social } \\
\text { para insultar o humillar a mi pareja o expareja. }\end{array}$ & 0,9 & 2,3 & 0,0 & 1,491 \\
\hline $\begin{array}{l}\text { Ha escrito un comentario en el muro de una red social } \\
\text { insultándome o humillándome. }\end{array}$ & 2,8 & 4,5 & 1,5 & 1,579 \\
\hline
\end{tabular}




\begin{tabular}{lllll}
\hline \multicolumn{1}{c}{ CDAQ } & $\begin{array}{c}\text { Total } \\
(\%)\end{array}$ & $\begin{array}{c}\text { Chicos } \\
(\%)\end{array}$ & $\begin{array}{c}\text { Chicas } \\
(\%)\end{array}$ & $X^{2}$ \\
\hline $\begin{array}{l}\text { He utilizado las nuevas tecnologías para hacerme pasar } \\
\text { por mí pareja o expareja y causarle problemas. }\end{array}$ & 0,9 & 2,3 & 0,0 & 1,491 \\
$\begin{array}{l}\text { Ha utilizado las nuevas tecnologías para hacerse pasar } \\
\text { por mí y causarme problemas. }\end{array}$ & 3,7 & 6,8 & 1,5 & 5,185 \\
$\begin{array}{l}\text { Ha amenazado, a través de las nuevas tecnologías, con } \\
\text { hacerme daño físicamente. }\end{array}$ & 1,8 & 2,3 & 1,5 & 2,156 \\
$\begin{array}{l}\text { Ha creado un perfil falso de mí en una red social con el fin } \\
\text { de causar problemas. }\end{array}$ & 0,9 & 2,3 & 0,0 & 1,491 \\
\hline
\end{tabular}

Nota. ${ }^{*} p<, 05 ;{ }^{* *} p<, 01 ;{ }^{* * *} p<, 001$

Diferencias de género en cuanto a la prevalencia de la perpetración y victimización de la violencia

En lo que se refiere a las diferencias de género (véase la tabla 1), solo se han encontrado diferencias significativas en «publicar música, poemas, frases, etc. en las actualizaciones de estado de las redes sociales, con la intención de insultar y/o humillar», en la perpetración, a favor de los chicos $(t=2,696 ; p \leq, 008)$. En cuanto a la victimización, también hay diferencias significativas de género en el mismo ítem, donde los hombres se ven más afectados que las mujeres ya que sus parejas realizan esta conducta $(t=2,098 ; p \leq, 036)$. En el resto de ítems las respuestas de chicos y chicas son similares.

\section{Posibilidad de padecer y ejercer abuso o maltrato}

Los datos obtenidos (véase la tabla 2) reflejan que, al parecer, las chicas tienen una percepción de riesgo mayor que los chicos ante la posibilidad de padecer abuso en una relación de pareja, suponiendo el $15,4 \%$ de las participantes, aunque no existen diferencias significativas entre chicos y chicas.

Tabla 2

Percepción de riesgo ante la violencia de pareja

\begin{tabular}{cccccc}
\hline \multicolumn{5}{c}{ ¿En qué medida crees que podrías padecer abuso o maltrato en una relación de pareja? } \\
\hline Nada (\%) & Algo (\%) & Bastante $(\%)$ & Totalmente $(\%)$ & $X^{2}$ \\
Chicos & 93,2 & 6,8 & 0,0 & 0,0 & 2,356 \\
Chicas & 84,6 & 12,3 & 1,5 & 1,5 & \\
\hline
\end{tabular}

Nota. ${ }^{*} p<.05 ;{ }^{* *} p<.01 ;{ }^{* * *} p<.001$

En cuanto a la posibilidad de ejercer abuso, los datos están bastante igualados entre chicos y chicas, con un $4,5 \%$ de los participantes (véase la tabla 3 ). 
Tabla 3

Posibilidad de ejercer abuso en una relación de pareja

\begin{tabular}{cccccc}
\hline \multicolumn{5}{c}{ ¿En qué medida crees que podrías ejercer abuso o maltrato en una relación de pareja? } \\
\hline \multirow{2}{*}{ Chicos } & Nada (\%) & Algo (\%) & Bastante (\%) & Totalmente $(\%)$ & $\mathrm{X}^{2}$ \\
Chicas & 95,5 & 4,5 & 0,0 & 0,0 & 0,831 \\
\hline
\end{tabular}

Nota. ${ }^{*} p<.05 ;{ }^{* *} p<.01 ;{ }^{* * *} p<.001$

\section{Conclusiones}

Los resultados apoyan estudios previos que también indican la exposición y realización de la violencia en la pareja joven (Fernández-González, 2013). Este trabajo estudia las conductas de victimización y perpetración de la violencia en una situación de noviazgo en población joven y analiza las diferencias de género que se pueden establecer en esta población, lo que contribuye a la literatura existente al respecto.

Respecto a los datos sobre victimización, un $18,3 \%$ de los participantes han sido victimizados por sus parejas a través de internet. Estos resultados arrojan la existencia de víctimas de violencia entre las personas jóvenes, en la línea de Durán y Martínez-Pecino (2015), aunque sea con menores prevalencias. Dichos autores obtuvieron porcentajes de victimización entre el $27 \%$ y el $57 \%$, aproximadamente. Sin embargo, en cuanto a la perpetración de la violencia, un 18,4 \% de los participantes afirma haber ejercido algún tipo de acoso a sus parejas a través de internet, dato que está en consonancia con este mismo estudio (Durán y Martínez-Pecino, 2015), donde se obtiene que un $14 \%$ ejerce acoso a través de internet.

En cuanto a las diferencias de género, en la línea de la misma investigación (Durán y Martínez-Pecino, 2015), hemos encontrado que los chicos $(21,12 \%)$ están más victimizados que las chicas $(15,52 \%)$. Aunque, por otra parte, chicos y chicas ejercen acoso en una medida similar, obteniendo porcentajes del $18,6 \%$ para los chicos y del 18,2 \% para las chicas, en contraposición con lo encontrado en dicho estudio. Más concretamente, las chicas demuestran, en mayor medida que los chicos, un mayor control sobre sus parejas, alcanzando cifras del $76,9 \%$ en comprobar la hora de última conexión de sus parejas, frente al $63,6 \%$ de los chicos; datos similares a los encontrados en otros estudios (Burke y cols., 2011). En cuanto a las diferencias, solo se han encontrado diferencias significativas en publicar contenidos en las redes sociales con el fin de humillar o insultar a su pareja, donde los chicos realizan esta conducta significativamente más que las chicas.

Los resultados obtenidos también ponen de manifiesto la bidireccionalidad de esta problemática, donde tanto chicos como chicas ejercen acoso hacia sus parejas en proporciones similares, apoyando aquellos estudios que describen la violencia entre las parejas jóvenes como un fenómeno bidireccional (Fernández-González, 2013; Straus y Luis, 2007; Straus y Mickey, 2012; Straus, 2004).

En conclusión, el elemento más prevalente de la violencia a través de internet parece ser el elemento de control, donde una gran proporción de los participantes realiza de forma casi rutinaria una supervisión de los comportamientos de su pareja a través de internet. Además, tanto chicos como chicas ejercen acoso hacia sus parejas a través de este medio.

Estos resultados cabría tenerlos en cuenta considerando algunas limitaciones. Por un lado, sería conveniente ampliar el número de participantes y las variables que podrían mediar 
en el problema. Además, se podría investigar si existen relaciones entre ser víctima y convertirse en un futuro acosador o viceversa. La identificación de factores que medien en la incidencia del problema abre caminos hacía la prevención de este tipo de situaciones.

En cualquier caso, el estudio ofrece aportaciones relevantes al analizar la violencia de pareja y el ciberacoso que se produce en la población joven, los cuales son fuertes usuarios de internet. A su vez, esto sugiere una modernización de los medios de violencia, dejando de lado la violencia física y adentrándose en la monitorización de la pareja, suponiendo un control mucho mayor.

\section{Referencias bibliográficas}

Archer, J. (2002). Sex differences in physically aggressive acts between heterosexual partners: A meta-analytic review. Aggression and Violent Behavior, 7, 313-351, en Fernández González, L. (2013). Prevención de la violencia en las relaciones de noviazgo: Aplicación y valoración de un programa para adolescentes. Universidad Autónoma de Madrid. Recuperado de https://repositorio.uam.es/bitstream/handle/10486/13026/62477_Fernandez Gonzalez Liria.pdf?sequence $=1$

Borrajo, E., Gámez-Guadix, M., Pereda, N. y Calvete, E. (2015). The development and validation of the cyber dating abuse questionnaire among young couples. Computers in Human Behavior, 48, 358-365.

Burke, S. C., Wallen, M., Vail-Smith, K. y Knox, D. (2011). Using technology to control intimate partners: An exploratory study of college undergraduates. Computers in Human Behavior, 27, 1162-1167.

Durán, M. y Martínez-Pecino, R. (2015). Ciberacoso mediante teléfono móvil e Internet en las relaciones de noviazgo entre jóvenes. Comunicar, 22, 159-167.

Fernández González, L. (2013). Prevención de la violencia en las relaciones de noviazgo: Aplicación y valoración de un programa para adolescentes. Universidad Autónoma de Madrid. Recuperado de https://repositorio.uam.es/bitstream/handle/10486/13026/62477_ Fernandez Gonzalez Liria.pdf?sequence $=1$

Finn, J. (2004). A survey of online harassment at a university campus. Journal of Interpersonal Violence, 19, 464-483.

Straus, M. A. (2004). Prevalence of violence against dating partners by male and femaleuniversity students worldwide. Violence Against Women, 10, 790-811.

Straus, M. A. y Luis, I. (2007). Gender Symmetry in Prevalence, Severity, and Chronicity of Physical Aggression Against Dating Partners by University Students in Mexico and USA. Aggressive Behaviour, 33, 281-290.

Straus, M. A. y Mickey, E. L. (2012). Reliability, validity, and prevalence of partner violence measured by the conflict tactics scales in male-dominant nations. Aggression and Violent Behavior, 17, 463-474.

Van Ouytsel, J., Van Gool, E., Walrave, M., Ponnet, K. y Peeters, E. (2016). Exploring the role of social networking sites within adolescent romantic relationships and dating experiences. Computers in Human Behavior, 55, 76-86.

Zweig, J. M., Dank, M., Yahner, J. y Lachman, P. (2013). The Rate of Cyber Dating Abuse Among Teens and How It Relates to Other Forms of Teen Dating Violence. Journal of Youth and Adolescence, 42, 1063-1077. 